\title{
Too "sexy" for the field? Paired measures of laboratory and semi-field performance highlight variability in the apparent mating fitness of Aedes aegypti transgenic strains
}

Andrew Aldersley ${ }^{*}$, Arissara Pongsiri ${ }^{2}$, Kamonchanok Bunmee $^{3}$, Udom Kijchalao $^{2}$, Wachiraphan Chittham², Thanyalak Fansiri ${ }^{2}$, Nattaphol Pathawong ${ }^{2}$, Alima Qureshi ${ }^{1}$, Laura C. Harrington ${ }^{4}$, Alongkot Ponlawat ${ }^{2}$ and Lauren J. Cator ${ }^{1}$

\begin{abstract}
Background: Evaluating and improving mating success and competitive ability of laboratory-reared transgenic mosquito strains will enhance the effectiveness of proposed disease-control strategies that involve deployment of transgenic strains. Two components of the mosquito rearing process, larval diet quantity and aquatic environment - which are linked to physiological and behavioural differences in adults - are both relatively easy to manipulate. In mosquitoes, as for many other arthropod species, the quality of the juvenile habitat is strongly associated with adult fitness characteristics, such as longevity and fecundity. However, the influence of larval conditioning on mating performance is poorly understood. Here, we investigated the combined effects of larval diet amount and environmental water source on adult male mating success in a genetically modified strain of Aedes aegypti mosquitoes in competition with wild-type conspecifics. Importantly, this research was conducted in a field setting using low generation laboratory and wild-type lines.
\end{abstract}

Results: By controlling larval diet (high and low) and rearing water source (field-collected and laboratory water), we generated four treatment lines of a genetically modified strain of Ae. aegypti tagged with fluorescent sperm. Laboratory reared mosquitoes were then competed against a low generation wild-type colony in a series of laboratory and semi-field mating experiments. While neither food quantity nor larval aquatic environment were found to affect male mating fitness, the transgenic lines consistently outperformed wild-types in laboratory competition assays, an advantage that was not conferred to semi-field tests.

Conclusions: Using a model transgenic system, our results indicate that differences in the experimental conditions of laboratory- and field-based measures of mating success can lead to variation in the perceived performance ability of modified strains if they are only tested in certain environments. While there are many potential sources of variation between laboratory and field lines, laboratory adaptation - which may occur over relatively few generations in this species - may directly impact mating ability depending on the context in which it is measured. We suggest that colony-hybridization with field material can potentially be used to mitigate these effects in a field setting. Release programs utilising mass-produced modified laboratory strains should incorporate comparative assessments of quality in candidate lines.

Keywords: Aedes aegypti, Mating success, Vector control, Body size, Larval conditioning

\footnotetext{
*Correspondence: a.aldersley@imperial.ac.uk

${ }^{1}$ Department of Life Sciences, Imperial College London, Silwood Park,

Ascot, UK

Full list of author information is available at the end of the article
} 


\section{Background}

Aedes aegypti is the primary vector of a range of arboviruses including dengue, chikungunya, Zika, and yellow fever, that affect the health of millions of people annually $[1,2]$. Recent efforts to control the spread of these diseases have focussed on the release of modified individuals to limit the reproductive or transmission capacity of local mosquito populations [3, 4]. Several such programs have now been extensively trialled [5-8]. In general, these strategies require released males to mate with wild females in the selected area to introduce a replacement or limiting mechanism into the target population [9]. The fitness of release lines is thus vital to program success; modified males not only must survive and disperse within the target environment, but also compete with wild conspecifics for mates [10-13]. One challenge to optimising male performance is understanding what key fitness parameters should be measured in the laboratory and how they relate to male fitness in the field [14]. Understanding and improving the performance of release males therefore has the potential to influence the operational success or failure of vector management initiatives.

All mosquito species are aquatic as juveniles [15]. Development of Ae. aegypti typically takes place in waterfilled containers found among households in urban and rural areas, such as discarded tyres, flower pots and buckets [16]. Larvae obtain food and nutrients from their surrounding habitat, feeding on plant matter and other organically-derived detritus [17]. Both the amount and content of food available affect larval development in Ae. aegypti. Variability in the quality of natural container environments is often high, resulting in heterogeneity in the physiological, nutritional, and energy status of the adult population [18-20].

As in many other invertebrate systems [21], larval conditioning has been linked to adult fitness in Ae. aegypti. Larval food quantity is one of the major determinants of adult body size [22], which is associated with a range of life history traits. Larger females are both more fecund and have a greater longevity [23, 24], while male size is positively correlated with sperm production and transfer $[25,26]$, survival [27], and increased pre-copulatory flight activity [28]. As larvae, mosquitoes also acquire a diverse microbiome from the rearing environment, which influences their development [29, 30] and adult physiology [31]. In addition, exposure to different microbes at the juvenile stage has recently been demonstrated to affect both body size [32] and egg production [33] in Ae. aegypti. Nutritional and microbial conditions experienced by larvae are known to interact with factors that drive vector competence in several medically important mosquito species [34-42]. In many insects, the microbiome governs adult behavioural processes [43]. However, the effect of the larval environment on adult mating competitiveness, a key aspect of mosquito fitness [10], is less understood.

This consideration is particularly relevant for those developing transgenic mosquito lines destined for release. The relationship between size and mating success in Aedes is unclear, with some studies indicating that small females preferentially mate with small males [44], while others have reported high copulation rates in large females regardless of male size $[26,45]$. In contrast to field-bred individuals, laboratory lines are often sustained under conditions intended to optimise developmental time and synchrony [44], generating cohorts that tend to be larger, and with a lower size variance, than wild populations [46]. Similarly, the role of the microbiome is often overlooked during production of release strains. Evidence from varied dipterans indicates that changes to the host microbiota can alter the sexual competitiveness [47-49] or mating preferences [50-52] of laboratory lines. These effects may be compounded by selective pressures introduced by prolonged laboratory rearing [53], which can result in habituation and reduced fitness in the field $[54,55]$. Thus far, studies into the mating success of transgenic or laboratory-reared Ae. aegypti have analysed factors such as male insemination capacity [25, $26,56]$, pre-copulatory signalling ability [45], and female fecundity $[23,57]$. Investigations of mating competitiveness in this species are limited. Most have applied laboratory-based assays $[28,58,59]$, while those utilising field approaches are relatively few $[60,61]$. It is not currently clear which quality control measures are most important for evaluating performance in mosquito release lines, yet larger-scale trials in natural conditions are considered an important tool in this process $[55,62,63]$.

Here, we tested the effects of larval diet amount and rearing water type on adult male mating success in a genetically modified laboratory strain of Ae. aegypti. We used an integrated approach combining laboratory and semi-field assays to assess several aspects of male mating fitness in competition with wild-type conspecifics in Kamphaeng Phet (KPP), Thailand: longevity, attendance to swarm sites, copulation and insemination success. We predicted that laboratory-sourced males fed on highquantity diets supplemented with water from naturally productive larval environments would enjoy a greater mating success against wild-type males than those fed on low-quantity diets or reared in laboratory water. We did not find evidence to support this prediction; male performance was not improved by increasing diet amount or by supplementing rearing environments with field-container water. However, males from the laboratory reared lines were found to outcompete wild-type conspecifics in laboratory mating assays. Importantly, laboratory-reared 
males did not display this advantage in semi-field cage trials. Our results suggest that adaptation to laboratory mating conditions can inflate the perceived mating success of release lines if they are only measured under laboratory conditions.

\section{Methods}

\section{Description of field site}

All experiments were conducted in the dengue endemic area of Muang District, Kamphaeng Phet (KPP) Province, Thailand, which is located approximately $360 \mathrm{~km}$ northeast of Bangkok. In 2018, there were 917 dengue cases and 2 deaths reported to the Ministry of Public Health in this region [64]. All four dengue serotypes have circulated in KPP [65]. The climate is tropical with three seasons: rainy (mid-May to mid-October), winter (mid-October to February), and summer (mid-February to mid-May) [66]. Investigations were performed at the Large Mosquito Enclosure semi-field facility constructed by the Department of Entomology, Armed Forces Research Institute of Medical Sciences (AFRIMS), Thailand in September and October 2018.

The AFRIMS site consists of three semi-field cages, $16.8 \times 125.5 \mathrm{~m}$ metal structures with a covered roof, semi-permeable mesh outer-walls, and dirt floors with sparse vegetation. The semi-field cages provide an enclosed space with a similar climatic profile to the natural habitat of Ae. aegypti. These outdoor facilities were observed to contain a number of common mosquito predators (geckos, spiders and ants). Within each semifield cage, we erected two further $6 \times 9 \times 3 \mathrm{~m}$ secondary containment areas constructed from bed-net material (Fig. 1a). These sub-enclosures were used to conduct semi-field mating competition and swarm site attendance, which are described in the sections detail below. Two BG-Sentinel traps (Biogents GmbH, Regensburg, Germany) baited with dry-ice and BG-Lure cartridge human odour were placed inside each semi-field cage in the area outside the sub-enclosures. These were left running constantly and monitored daily during experiments to estimate the escape-rate from the secondary containment cages. Mosquitoes captured in these traps were not included in analyses. All other experimental work was performed in adjacent temperature-controlled laboratory buildings on site. Climatic conditions were monitored in all rearing environments, laboratories, and the semifield cages using a combination of EasyLog USB (Lascar Electronics Ltd, Whiteparish, Wiltshire, UK) and HOBO (Onset Computer Corporation, Bourne, MA, USA) data loggers. Temperatures averaged $30.7 \pm 5.3{ }^{\circ} \mathrm{C}$ in semifield cages over the duration of the test period, with a relative humidity of $74.5 \pm 17.6 \%$.

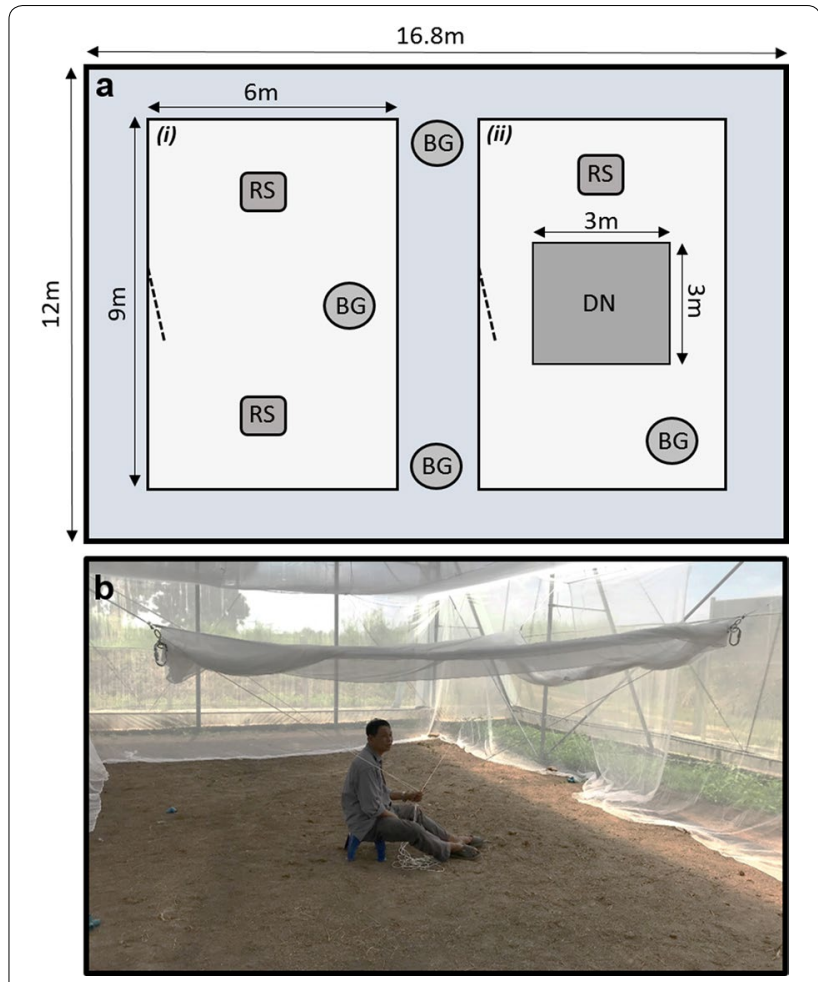

Fig. 1 Layout of individual semi-field cages. a Two sub-enclosures constructed from bednet material were erected inside each semi-field cage structure. These had entrance points on the left-side (dashed line). The leftmost sub-enclosure ( $i$ ) was used for mating competition assays, while the right (ii) was used for swarm site attendance experiments. In (ii), a drop-net (DN) was hung and used to capture males attracted to the host. BG-Sentinel traps (BG) were left inside the sub-enclosures and in the main semi-field cage area for mosquito recapture. Resting sites (RS), partially filled with water, were also placed inside each experimental arena. Access to the semi-field cages was controlled by a double sliding door. b Drop-net procedure for measuring swarm site attendance inside a semi-field cage sub-enclosure. Host is positioned under the raised drop-net, which is released after $10 \mathrm{~min}$. Male mosquitoes in close proximity to the host are trapped and recaptured using a vacuum aspirator

\section{Survey and collection from natural habitats}

We surveyed domestic sites in KPP for active water storage containers (sampling dates: 10th, 16th and 17th September, and 1st and 2nd October 2018). A container was considered to be active when at least 10 fourth-instar Aedes mosquito larvae and/or pupae (identified using physiological indicators [67]) were easily observed inside it. We collected water (a minimum of $0.5 \mathrm{l}$ ), debris, larvae, and pupae from a total of 21 containers. Samples were strained of large organic matter and living organisms and combined into a field-container mixture used during rearing of experimental lines (see below). This was supplemented with rainwater collected at the field laboratory site when necessary. We used this homogenised blend of container samples to represent the 
microbial community present in natural breeding sites of Ae. aegypti in KPP.

\section{Mosquito lines and rearing}

A laboratory strain of Ae. aegypti mosquitoes homozygous for the fluorescent protein DsRed [68] was produced from a cross between transgenic Higgs' White Eye (HWE) DsRed mosquitoes and a third generation field line originating from KPP (DsRedKPP line, details in Additional file 1: Text S1). We subjected this line to treatment conditions that manipulated both the larval rearing water type (laboratory or field-container water) and the quantity of food they were offered (Cichlid Gold, Hikari, Kyrin Food Industries Ltd, Japan): high $(1.0 \mathrm{mg} /$ larvae/day) or low $(0.3 \mathrm{mg} /$ larvae/day ground fish food); details of the diet selection process are given in Additional file 1: Text S2. This regime yielded adults from four different diet-water treatment combinations: High-Lab (HL); Low-Lab (LL); High-Field (HF); and Low-Field (LF). DsRedKPP eggs were submerged in mains-supplied tap water from the field laboratory and left for a maximum of $24 \mathrm{~h}$ to induce hatching. First-instar larvae were separated into plastic trays $(33 \times 23 \times 9 \mathrm{~cm})$ containing $2 \mathrm{l}$ of water and reared at a density of 250 larvae/l. Larvae from the HL and LL treatments were reared in laboratory tap water (as above). In the HF and LF treatments, larval trays were filled with 1.61 of laboratory tap water and $400 \mathrm{ml}$ of the field-container mixture. Trays were covered with netting and stored under ambient conditions $\left(29.6 \pm 2.9^{\circ} \mathrm{C}\right.$ and $76.0 \pm 11.5 \%$ relative humidity over the duration of the rearing period) on shelving units inside a small windowed building.

A competitor line of KPP wild-type Ae. aegypti (KPPWT line) was established by sampling larvae (c.2000) from villages in 3 different subdistricts of KPP in July 2018. These larvae were mixed in trays (as described above) filled with field-container water and allowed to eclose. Adults were sorted to retain only Ae. aegypti, which were mixed in cages $(30 \times 30 \times 30 \mathrm{~cm})$, offered a $10 \%$ sucrose solution, and permitted to mate. Females (c.800) were given a human blood meal and allowed to oviposit, from which we obtained first generation offspring. These individuals were hatched in large black plastic containers $(44 \times 34 \times 17 \mathrm{~cm})$ filled with a mixture of laboratory tap water (14 l), rainwater collected from the field laboratory site (1.6 l), and the field-container mixture $(400 \mathrm{ml})$. Trays contained approximately 1000 larvae. On each day, $400 \mathrm{ml}$ of water was decanted from the rearing trays and replaced with a fresh sample of the field-container mixture. From the fourth day after hatching, we supplemented the available nutrients in the trays daily with $6 \mathrm{mg}$ of ground fish food. The KPPWT trays were also covered with netting and stored outside in the shade $\left(29.3 \pm 3.8{ }^{\circ} \mathrm{C}\right.$ and $29.7 \pm 8.7 \%$ relative humidity over the duration of the rearing period).
Upon pupation, individuals from each treatment were transferred into individual tubes. Newly eclosed adult males and females were placed into separate cages $(30 \times 30 \times 30 \mathrm{~cm})$ for each treatment condition and offered a $10 \%$ sucrose solution. All adult cages were stored in a temperature-controlled laboratory building $28.4 \pm 1.5{ }^{\circ} \mathrm{C}$ and $28.3 \pm 4.2 \%$ relative humidity over the course of the test period). To monitor and control adult ages throughout experiments, new cages were established every 2 days.

\section{Nutritional analyses}

We conducted nutritional analyses (MR4 protocol adapted from [69-72]) on a subsample of freshly eclosed males from the four DsRedKPP treatments (LL, LF, HL and HF) and the KPPWT line used in experiments. In addition, we also ran analyses on pupae collected from six of the productive containers identified during the field survey. Sugar, lipid, and glycogen stores for each male were quantified using a colorimetric method.

\section{Experimental design}

We performed a minimum of two replicates for each of the assays described below. These replicates were conducted in three blocks due to the availability of three semi-field cages at the field laboratory site. Blocks were staggered such that they successively overlapped, beginning every $4-5$ days (full details of the treatments included in each block are given in Additional file 2: Table S2). KPPWT mosquitoes were hatched periodically every 3 days to ensure a regular supply of appropriately-aged adults was available for each blocked experiment. To prevent cross-contamination across blocks, we ensured a break of at least $24 \mathrm{~h}$ between experiments conducted inside the semi-field cages. Furthermore, adults from the different treatments were rotated across all semi-field cages both between (mating competition assay) and within (swarm site attendance assay) blocks, to account for any variation in semi-field cage microenvironments that could have affected experimental outcomes.

\section{Adult survival assay}

We recorded the adult survival of a subset of individuals from the High-Lab (HL), Low-Lab (LL) KPPWT lines. We took 10 males from each treatment (less than 1-day from eclosion) and placed them together into a small cup $(0.45 \mathrm{l})$. These were then offered either a $10 \%$ sucrose solution ( $n=2$ containers per treatment) or water only ( $n=5$ containers per treatment) soaked into cotton wool. Adults were held inside under ambient conditions $\left(30.3 \pm 2.4{ }^{\circ} \mathrm{C}\right.$ and $69.8 \pm 7.5 \%$ relative humidity). Mortality was monitored in each container daily. 


\section{Laboratory mating competition}

We investigated the impact of larval diet on male mating response and success when in direct competition with wild-type individuals. Following previous methodology [28], two 2-6 day-old virgin males from one of the four diet-water treatments (DsRedKPP) were competed against two 2-4 day-old virgin KPPWT males for a single 2-4 day-old virgin wild-type female (KPPWT). A subset of males from each treatment condition was selected at random and marked with either yellow (DsRedKPP strains) or pink (KPPWT strain) dust (Swada Inc, Stalybridge, Cheshire, UK) [28, 73], then left overnight to recover. The following day, males were aspirated into a cylindrical container $(2.83 \mathrm{l})$ and left for $5 \mathrm{~min}$ to acclimate. A single KPPWT female was then introduced into the cage. Host cues were provided by proximity of the investigator. The individuals were left undisturbed under observation until formation of the first copula (defined as genital contact for a minimum of $5 \mathrm{~s}$ ), at which point the mating pair was aspirated from the cage (whilst still coupled). The strain of the successful male (DsRedKPP or KPPWT) was identified by inspection of dust colour. This assay was repeated 20 times in two replicates for each diet-water treatment. The right wings of the females and successful and unsuccessful males from these experiments were dissected and measured to estimate body sizes [74] across the treatment groups.

\section{Semi-field cage mating competition assay}

We measured the effect of larval rearing treatment on male mating success under semi-field conditions. To begin, 100 2-5 day-old virgin KPPWT females were released into one of the semi-field cage sub-enclosures (Fig. 1a) between 7:00 and 7:30 h, and allowed to naturally disperse and settle. At either end of the sub-enclosure were positioned two resting sites consisting of an open-topped black-cloth covered box $(35 \times 35 \times 55 \mathrm{~cm})$ with a partially-filled bucket of water placed inside (Fig. 1a). Approximately 60 min later, $1003-7$ day-old virgin DsRedKPP males taken from one of the four larval treatments were released into the sub-enclosure, along with 100 2-5 day-old virgin KPPWT males. Males were released simultaneously from separate containers placed next to one another at the rear-end of the subenclosure. Mating was allowed to occur in the enclosure for 10-11 hours. Host stimuli were provided by walking around the edges of the cages in the morning and lateafternoon of the release day. Starting between 18:00 and 18:30 h, surviving individuals were collected back using a BG-Sentinel trap located inside the sub-enclosure (Fig. 1a). The following morning and evening, we entered the sub-enclosures and collected any remaining mosquitoes using a prokopack [75] or CDC vacuum aspirator.
The BG-Sentinel trap was left running for $36 \mathrm{~h}$ and checked twice-daily to maximise mosquito recapture.

Collected females were immediately sedated with cold and dissected in PBS to determine their mating status. The presence of sperm in the spermathecae confirmed successful insemination. We used a DsRed filter attachment (ET-CY3/TRITC, Chroma Technology Corporation, Vermont, USA) on a stereomicroscope (BX50 Fluorescence Microscope, Olympus Corporation, Tokyo, Japan) to visualise florescent sperm in the spermathecae and infer whether the female was mated by a male from the DsRedKPP (positive DsRed signal) or KPPWT (negative DsRed signal) strain. Mating success was defined as the proportion of recaptured females that were successfully inseminated by released males from either respective treatment. We also counted the number of recaptured males to provide an overall estimate of mortality rates across the semi-field cages.

\section{Swarm site attendance}

We tested the effect of larval rearing condition on adult male attendance at swarm sites inside the semi-field environment. Ten 2-6 day-old virgin male KPPWT and DsRedKPP mosquitoes (from one of the four diet-water treatments) were dusted with either pink (KPPWT) or yellow (DsRedKPP) dust and left overnight to recover. The following morning, between 7:00 and 8:00 $\mathrm{h}$, the males were released into the relevant sub-enclosure within one of the semi-field cages (Fig. 1a). This contained a weighted mesh canopy drop-net $(3 \times 3 \times 2 \mathrm{~m})$ with a quick release drawstring positioned in the centre (Fig. 1b), fixed in the raised position as the males were released. Each sub-enclosure also contained a partially covered and water-filled black bucket (diameter: $30 \mathrm{~cm}$; height: $40 \mathrm{~cm}$ ) resting site (Fig. 1a). After one hour of acclimation, a single human host entered the sub-enclosure and walked around the perimeter once. They then positioned themselves under the raised drop-net for a period of $10 \mathrm{~min}$. At this time, the drop-net was released capturing any males in close proximity to the host. Males trapped within the net were then collected using either a prokopack [75] or CDC vacuum aspirator. We also separately collected all males that were not trapped in the drop-net (i.e. those that were unresponsive to the host) with the use of vacuum aspirators and a BG-Sentinel trap, which was left running inside the sub-enclosure until 18:00 h.

Recaptured males were counted and the numbers collected from each treatment (KPPWT or DsRedKPP) determined by dust-colour verification. The proportion of males from each group that attended the swarm site was determined by dividing the number found within the drop-net by the total number recaptured. This assay was 
repeated with a new set of males three times in two replicates for each diet-water treatment $(n=6$ trials per treatment). A total of 13 dusted males (from 480 released) were caught in traps placed outside the semi-field cage sub-enclosures (Fig. 1a). These individuals were not included in our analysis but confirmed that the escaperate from the sub-enclosures was low $(<3 \%)$.

\section{Statistical analyses}

All statistical tests were run using the $\mathrm{R}$ software environment [76] using a combination of packages car [77], lsmeans [78], lme4 [79], multcomp [80], dplyr [81] and coxme [82]. To test whether larval diet-water treatment had an effect on adult male body size, we used an ANOVA model of wing length data with larval diet amount (high or low), rearing water type (laboratory or field), experimental replicate (1 or 2 ), and their interactions. All post-hoc comparisons were made using Tukey's HSD test with a Bonferroni correction to account for multiple contrasts. We tested the effect of larval water source and diet amount on the abundance of energy reserves of newly emerged males using a general linear model (GLM). Post-hoc comparisons between treatment groups were made using Tukey's HSD test with a Bonferroni correction. Mann-Whitney U-tests were then used to compare lipid, glycogen and sugar levels between the KPPWT and field-collected strains. We investigated the effect of larval diet treatment and adult diet on adult mortality using censored Cox proportional hazards models. Post-hoc pairwise comparisons between separate treatments were made using log-rank tests [83] with $P$-values adjusted where appropriate using the Bonferroni correction to account for multiple contrasts. To investigate the overall relative success of DsRedKPP males compared to the KPPWT line in small-cage mating competition experiments we used Pearson's chi-square test. A mixed effects binary logistic regression model (GLMM) was used to explore the effect of larval diet-water condition on mating competition outcome (whether the successful male was from the DsRedKPP or KPPWT line). Larval treatment (HL, HF, LL or LF) was included as a fixed effect, while replicate and block were added as random effects.

In the semi-field cage mating competition assay, we used a weighted binomial mixed effects regression model (GLMM) to explore the effect of larval diet-water condition on DsRedKPP male mating success. The proportion of DsRedKPP-mated females, weighted by the total number of females collected, was modelled as a function of larval treatment (HL, HF, LL or LF), with replicate and semi-field cage identity included as random effects. The number of males attending swarm sites across the two mosquito strains used (DsRedKPP and wild-type) was assessed using a Kruskal-Wallis rank sum test. To investigate whether larval diet-water treatment had an effect on the proportion of males attending swarm sites, we used a weighted binomial mixed-effects model (GLMM). The proportion of DsRedKPP males caught inside the dropnet, weighted by the total recaptured, was modelled as a function of larval condition (HL, HF, LL or LF), with replicate included as a random effect. Differential recapture rates in the semi-field experiments were assessed using separate Poisson mixed effects models. Either the total number of females (mating competition) or males (swarm site attendance) collected were included as the response variable, with semi-field cage identity and larval treatment included as fixed effects, and replicate and experimental block as random effects. Post-hoc pairwise comparisons (across semi-field cages) were performed using Tukey's HSD test with a $P$-value correction.

\section{Results}

\section{Adult body size and nutrition}

Manipulation of rearing conditions led to differences in the adult body sizes and nutritional reserves of males from different larval treatments (Fig. 2, Additional file 2: Table S3, Additional file 3). The interaction between food amount and rearing water type significantly affected body size (ANOVA: $F_{(1,578)}=25.20, P<0.0001$ ). High diet treatments produced males that were larger than low or wild-type conditions, but which were not significantly different from one another. At low larval diets, individuals reared in field-container water were found to be significantly smaller than those from laboratory water, which

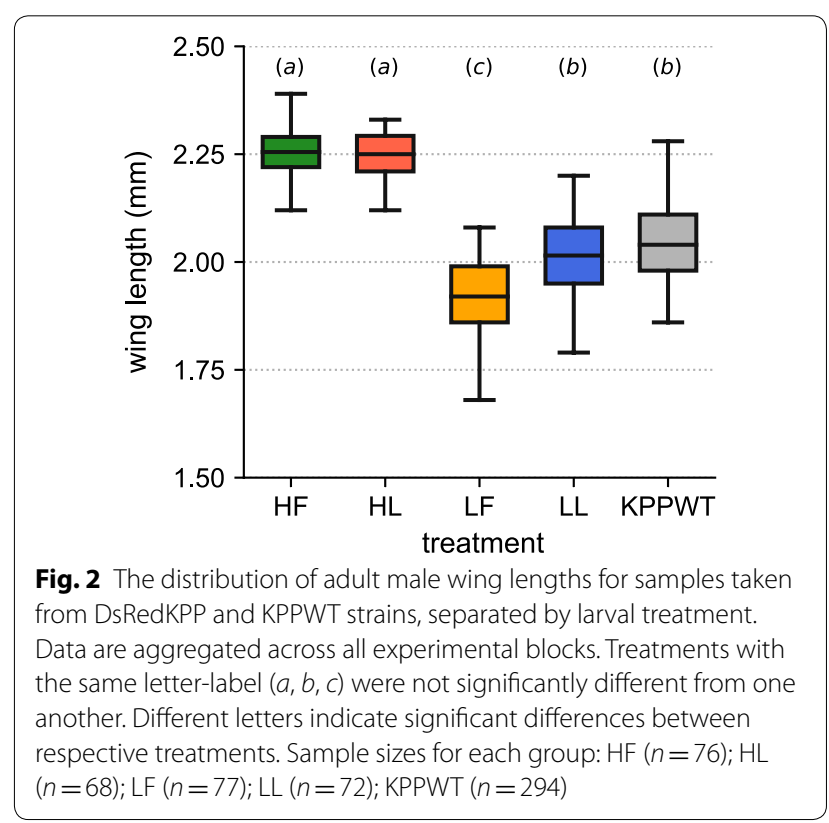


themselves were not different in size from the KPPWT strain. Furthermore, we detected a significant replicate effect within treatments (ANOVA: $F_{(1,578)}=12.88$, $P<0.0001$ ), which was found to affect males from the LF and KPPWT conditions only (Additional file 2: Figure S2). This did not, however, influence the overall size-trend between treatments across blocks.

Males from the high diet treatments were found to have a greater lipid content than those from low diet treatments (GLM: $\left.F_{(2,43)}=32.59, P<0.0001\right)$, but did not differ in terms of their glycogen or sugar reserves. Rearing water source had no significant effect on nutritional abundance (GLM; lipids: $F_{(1,28)}=0.17, P=0.69$; glycogen: $F_{(1,47)}=2.22, P=0.14$; sugar: $\left.F_{(1,46)}=0.17, P=0.69\right)$. The KPPWT males had a nutritional profile similar to those from the DsRedKPP LL and LF treatments (Additional file 2: Table S3), with a significantly lower stored lipid amount than the HF treatment group (Additional file 2: Table S3, Tukey's HSD test; $P<0.0001$ ), and marginally significantly less than HL (Additional file 2: Table S3, Tukey's HSD test; $P=0.06$ ). When compared to field-collected males, those from the KPPWT strain had similar lipid stores $(54.77 \pm 18.48$ vs $59.10 \pm 8.70 \mathrm{mg}$; Mann-Whitney U-test; $U=29.50, \quad n=16, \quad P=0.52)$, but a significantly higher glycogen $(66.78 \pm 22.00$ vs $34.64 \pm 27.99 \mathrm{mg} ;$ Mann-Whitney U-test; $U=94.00$, $\left.n=21, \quad P=3.00 \times 10^{-3}\right)$ and sugar $(31.44 \pm 12.09$ vs $18.64 \pm 7.79 \mu \mathrm{g}$, Mann-Whitney U-test; $U=88.50, n=21$, $P=0.012)$ content.

\section{Adult survival assay}

As expected, overall mortality rates (Additional file 3) were higher for males offered a water-only diet (Fig. 3a) in comparison to those held on 10\% sugar solution (Fig. 3b). Larval diet amount was found to be a significant factor in determining individual survival probabilities when adult males were sustained on both the water-only (Cox Proportional Hazards model log-rank test; $\chi^{2}{ }_{(2)}=186.25$, $P=3.78 \times 10^{-41}$ ) and sugar-based (Cox Proportional Hazards model log-rank test; $X^{2}{ }_{(2)}=6.25, P=4.39 \times 10^{-2}$ ) diets. When sustained on water only, males from the high-diet (HL) condition had a significantly greater survival likelihood than those from the wild-type strain (KPPWT), which in turn survived significantly longer than low-diet (LL) individuals (post-hoc pairwise tests with Bonferroni correction; $P<0.05)$. There were no significant mortality differences between larval treatments on the sugar-based diet (post-hoc pairwise tests with Bonferroni correction; $P>0.05$ ).

\section{Laboratory mating competition}

DsRedKPP males were more likely to form a copula with a virgin wild-type female in laboratory assays (Fig. 4a,

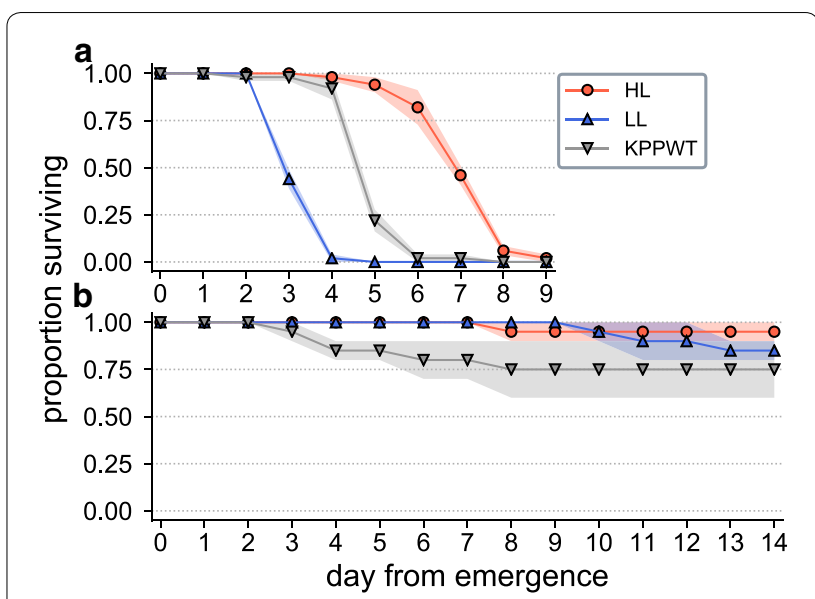

Fig. 3 Adult male survival of individuals from different larval treatments offered a diet of either water (a) or 10\% sugar solution (b). Data plotted show the mean across all replicates $(n=5$ per treatment for water; $n=2$ per treatment for sugar), with shaded regions indicating the standard error

Pearson's chi-square test; $\chi^{2}{ }_{(1)}=22.50, P=2.10 \times 10^{-6}$ ) and were successful in $68.8 \%$ of matings when in competition with the KPPWT strain (Additional file 3). The specific diet-water treatment that larvae were reared on was not, however, found to affect relative mating success between the groups (GLMM; $P>0.05$ for all treatment levels).

\section{Semi-field cage recapture rates}

We investigated individual recapture rates in semi-field assays to determine potential sources of bias within the data collected. During semi-field mating competition experiments, female recapture rates were significantly affected by the semi-field cage used (Table 1, type-III ANOVA run on GLMM; Wald $\left.\chi^{2}=9.53, P=8.54 \times 10^{-3}\right)$. Recapture rates were significantly greater in cage 2 than either cage 1 or 3 (Table 1). This pattern was weakly mirrored in the swarm site attendance tests (Table 1). However, the number of males collected did not differ by strain (DsRedKPP vs KPPWT, Kruskal-Wallis test; $X^{2}=0.30, P=5.81 \times 10^{-1}$ ), and neither larval treatment nor the particular semi-field cage used were found to significantly affect the total recapture count in this assay (type-III ANOVA run on GLMM; $P>0.50$ for both factors). Given the low escape rates observed $(<3 \%)$, this suggests an overall mortality rate between $10 \%$ and $40 \%$ within the semi-field cages.

\section{Semi-field cage mating competition}

The proportion of recaptured females that were found to be mated was consistently high $(>92 \%$, Additional file 2: Table S4, Additional file 3). Of these, an average 

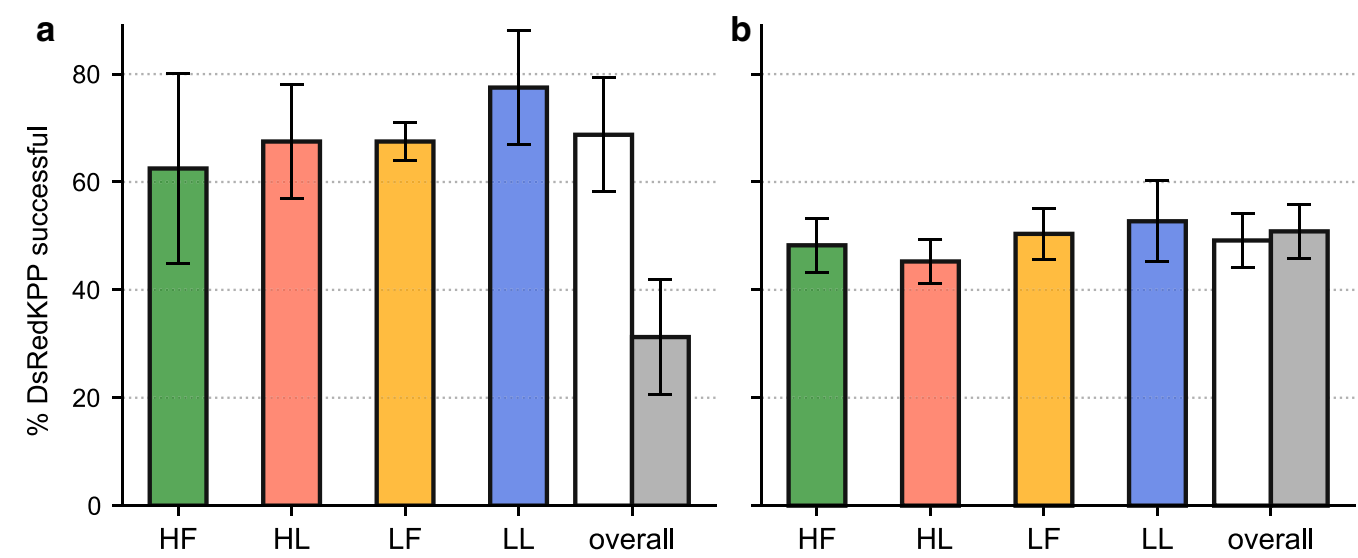

treatment group

Fig. 4 Results from the small cage and semi-field mating experiments. a The mean ( \pm standard deviation) proportion of trials in which a DsRedKPP male was the first to copulate with a KPPWT female in small cage mating competition assays. b The mean ( \pm standard deviation) proportion of mated recaptured females that were found to be inseminated by DsRed-tagged sperm in semi-field mating assays. Data are averaged across each replicate and separated by larval treatment. For each graph, the "overall" treatment group shows the DsRedKPP success rate averaged across all treatments (white bars) versus the corresponding average KPPWT success rate (grey bars)

Table 1 Mean ( \pm standard deviation) total number of released adult females and males that were recaptured in, respectively, the semi-field mating competition and swarm site attendance assays, separated by cage number. Data are calculated across all trials performed within the given semi-field cage

\begin{tabular}{lll}
\hline Cage & $\begin{array}{l}\text { o recaptured, mating } \\
\text { competition (\%) }\end{array}$ & $\begin{array}{l}\text { o recaptured, } \\
\text { swarm site } \\
\text { attendance (\%) }\end{array}$ \\
\hline 1 & $53.5 \pm 2.12$ & $68.8 \pm 11.88$ \\
2 & $81.0 \pm 2.65^{*}$ & $76.9 \pm 16.24$ \\
3 & $61.3 \pm 7.09$ & $70.0 \pm 16.04$ \\
\hline *Indicates significant difference with $P<0.0001$ from pairwise Tukey's HSD tests
\end{tabular}

of $49.2 \%$ were identified as having been inseminated by males from the DsRedKPP strain (Fig. 4b, Additional file 2: Table S4). Larval condition was not found to significantly affect male mating success in these assays (GLMM; $P>0.50$ for all conditions).

\section{Swarm site attendance}

The mean number of males collected inside the dropnet was $5.63 \pm 1.95$, giving an average swarm site attendance rate of $39.53 \pm 11.28 \%$ (Additional file 3 ). The number of males attending swarm sites did not vary by mosquito strain (DsRedKPP $v s$ wild-type, Kruskal-Wallis rank-sum test; $P>0.50)$. Furthermore, when variation due to replicate effects was controlled for, swarm site attendance did not differ by diet-water treatment (Fig. 5, type-III ANOVA run on GLMM; $P>0.10$ for all treatment levels).

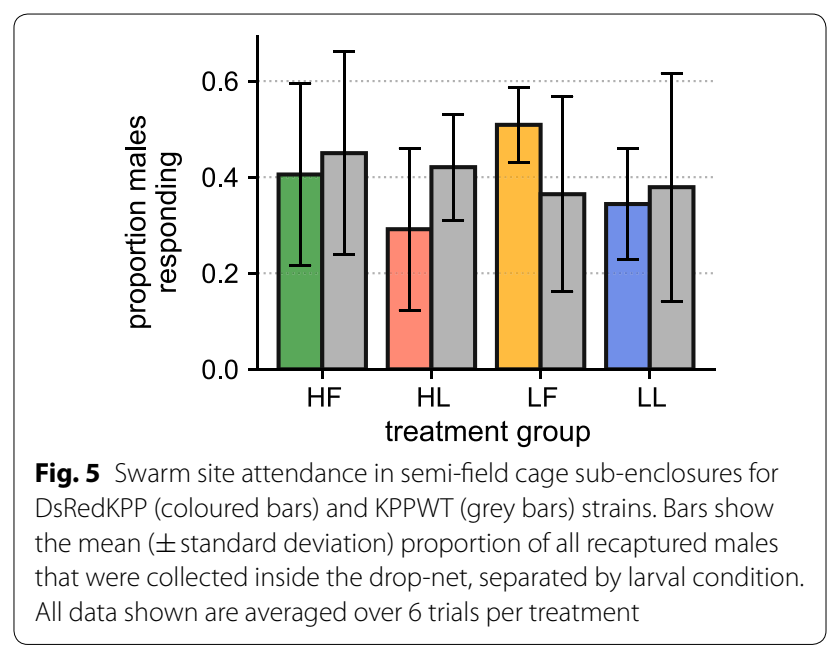

\section{Discussion}

The mating success of mosquito lines intended for release is a key consideration for the design of population control programs intended to reduce disease transmission. Importantly, the ability of transgenic males or those infected with Wolbachia or other sterilizing/transmission blocking bacteria to locate and compete for wild conspecifics in natural habitats is a fundamental aspect of their fitness [10]. While larval condition is known to influence various life history traits in mosquitoes, its effect on adult mating performance is not fully understood.

Here, we used a combination of laboratory and semifield assays to investigate the joint effects of larval 
nutrition and aquatic environment on the mating success of a genetically modified strain of Ae. aegypti in direct competition with a wild-type line sourced from Kamphaeng Phet (KPP), Thailand. Variation in larval rearing condition did not influence male mating success across the treatments used. Neither increasing the amount of food available to larvae nor supplementing their aquatic habitat with field-container water improved performance relative to the wild-type strain. However, we observed a large discrepancy between laboratory and field-based measures of mating fitness, with transgenic males performing significantly better than wild-types in laboratory assays, an advantage that was not observed in the semifield system. These findings suggest that differences in experimental designs can alter the perceived quality of lines if they are only measured under particular conditions. It is thus possible that mosquito strains intended for use in control programs, whose genetic, behavioural, and rearing backgrounds (including the potential effects of adaptation to laboratory environments $[54,55])$ are often quite different to members of the target population into which they are released, could have a higher estimated performance ability if they are only assessed in laboratory settings. Importantly, our results highlight the need to incorporate field-based measurements of mating performance as part of control evaluations.

Variation in adult male body size between larval treatment groups was predominantly driven by food quantity (Fig. 2) [46]. Cohorts fed lower diet amounts had a smaller average body size, but greater size variance, than those in the high-diet groups, which yielded males of similar proportions (Fig. 2). Reduced food quantity results in staggered emergence of adult Ae. aegypti [84] and may alter underlying physiological mechanisms of development that are dependent on environmental factors (such as temperature [22]). Climatic conditions varied drastically over the rearing period. The interaction of nutritional level with other controls on larval growth may account for our observation of significant differences in the body sizes of males reared on low level diets across experimental blocks. It is also possible that other determinants of mosquito development, such as the structure of the gut microbiota [29], interact with food quantity to influence adult morphology. Mosquitoes from different rearing environments harbour distinct bacterial communities [30], and those raised under laboratory protocols are likely dissimilar to natural field populations [85]. Interestingly, we note that only lower diet, field-container water treatments (LF and KPPWT) displayed a significant size-replicate effect, indicative of a high variation between rearing cohorts that was not present in high diet and laboratory water conditions (Additional file 2: Figure S2). The interactions between microbiome, nutrition, and environment in determining adult mosquito physiology are likely both highly sensitive and complex, and further work is required to understand their relative roles in developmental processes.

Our findings support the notion that male mating performance in Aedes is more nuanced than "bigger is better" [45], as appears to be the case for Anopheles mosquitoes [86, 87]. Recent analyses of mating in Ae. aegypti have indicated that success may be influenced by a relative size-assortative relationship [44, 45]. Size-assortative mating could be driven by the mate-grasping mechanics of Aedes copulation, which requires males to seize and orient venter-to-venter with females using their legs in order to facilitate genital contact [88, 89]. As a result, it has been suggested that release lines may enjoy greater mating success if their body size distribution more closely matches that of the target field group [44, 45]. While our results did not reveal a significant effect of larval diet quantity (and therefore body size, Fig. 2) on male mating competitiveness, we note that DsRedKPP males reared on a low larval diet quantity (which had a larger size overlap with the competitor KPPWT line) were observed to perform marginally better in mating assays than those from high diet conditions. Establishing the strength of this effect, and how it may contribute towards the potential performance of release lines in the field, is a worthwhile avenue for future investigation.

During laboratory-based small-cage mating trials, males from the DsRedKPP line were consistently more successful in being the first to form a copula with a KPPWT female, no matter what their larval condition (Fig. 4a). This is potentially a consequence of adaptation of the DsRedKPP strain to mating in such confined enclosures during colonisation and laboratory maintenance (see Additional file 1: Text S1). Competitive selective pressures are thought to be high in such rearing environments. Recent experimental evidence indicates that evolutionary responses in mating performance can emerge in as little as five generations in this species [90]. Practically, this would confer a greater advantage to the DsRedKPP males during laboratory mating competition assays, which may explain the discrepancy between the measures of sexual fitness that we observed. However, it is important to note that differences between the mating performance of the DsRedKPP and KPPWT lines may also be attributable to variation in other genetic and rearing factors between the strains. Establishing the effects of laboratory adaptation on mosquito performance is currently an active field of investigation [54], and further work is required to understand its implications for mating fitness in mass-produced lines.

In the semi-field assays, DsRedKPP males performed comparably to KPPWT individuals no matter which 
larval treatment they came from. Males from the laboratory strain showed similar levels of swarm site attendance (a proxy for their attractiveness towards mating arenas, Fig. 5) and achieved a comparable rate of female insemination in large-scale mating trials (Fig. 4b) when compared to their wild-type counterparts. While we were not able to detect specific instances of multiple female insemination (mating of a single female by both a DsRedKPP and KPPWT male), rates of polyandry in semi-field colonies of Ae aegypti are known to be low [91], and unlikely to substantially alter the results presented here. Furthermore, and in contrast to observations from previous investigations of laboratory mosquito strains [92], the males used in this study appeared to respond appropriately to swarming cues (Fig. 5). Under natural conditions, therefore, there appears to be little impact of laboratory habituation or genetic modification on male mating competitiveness, a finding that corroborates reports elsewhere from studies of Aedes mosquitoes [56, 58, 59, 61, 93-95]. The DsRedKPP strain used here originated from a cross between a long-term laboratory colony and a low generation line (see Additional file 1: Text S1). There is evidence that reproductive phenotypes in laboratory-adapted mosquito strains can be restored, at least partially, to wild-type levels by crossing between lines [63]. Our results suggest that where laboratory-induced habituation costs on mating behaviours do exist, they can potentially be mitigated through colony-hybridization. The relative benefits of outbreeding transgenic strains with laboratory [63] and field [53] material on mating performance warrants deeper consideration.

Standardised assessments are now recognised as a requirement to determine the quality of release strains prior to deployment in the field [14,96-98]. While in this study we used a model transgenic system not intended for use as part of any mass-release program, our results nevertheless demonstrate that different approaches to measuring mating success can produce quite different outcomes. These findings highlight the importance of testing different fitness measures across a range of environments; success in laboratory settings does not necessarily translate to high performance in natural scenarios.

\section{Conclusions}

Our findings show little effect of larval diet and rearing environment on adult sexual competitiveness of a transgenic line when compared to wild-type counterparts, but offer several important insights for the development and testing of mass-reared lines. First, we highlight the discrepancy between laboratory-based and field-based metrics of mating success. Laboratory assays may present conditions advantageous to habituated strains, which taken alone, could lead to an overestimation of their performance in field situations. We stress the importance of validation between approaches used to assess the quality of different release lines; further work is required to determine specific fitness effects on varied transgenic systems developed for use in unique mosquito control programs. Secondly, it has been suggested that optimising the fitness of release strains may fundamentally rely on a trade-off between characteristics. For instance, selecting for larger males, which our results show to be positively correlated with longevity (Fig. 3) [28], and which has previously been associated with increased sperm transfer $[25,26]$, could improve male opportunities to mate in low density field environments [99]. However, previous work indicates that this may come at a cost of reduced sexual competitiveness due to a size-mismatch with the target population $[44,45]$. Our results do not support the existence of a strong size-assortative mating effect in semifield mating assays, yet further work is required to fully explore the potential implications of body size on release line fitness in natural conditions. Finally, while we do not find clear evidence for an influence of larval condition on adult male mating success, we cannot rule out the possibility that diet and rearing water source may impact field performance after long-term selection. Recommendations to maintain the fitness and mating competitiveness of laboratory insect strains destined for controlled release include diet variation and supplementation, and frequent backcrossing with wild-types to promote an outbred genetic background [53]. Ultimately, this requires both a detailed knowledge of developmental and physiological characteristics of the target population, coupled with a better understanding of mosquito mating biology. Taken together, these efforts have the potential to drastically improve mosquito control technologies.

\section{Additional files}

Additional file 1: Text S1. Supplementary methods detailing generation of the DsRedKPP strain. Text S2. Determination of high and low laboratory diet quantities via a preliminary pilot study. Figure S1. Weighted larval development and pupation rates across pilot diet treatments, plotted as a function of time (from hatch). a Mean daily instar ( \pm standard error) of all remaining larvae (i.e. those which had not pupated or died). b Total number of pupae counted each day. Table S1. Wing lengths of adult males from different diet treatments ( $n=12$ per treatment).

Additional file 2: Table S2. Summary of experimental blocking design. Table S3. Nutritional content analyses of mosquitoes in experimental treatment groups. Figure S2. Wing lengths of adults from all larval treatments, separated by experimental block. Table S4. Full results of semifield cage mating competition experiment.

Additional file 3: Dataset S1. Supporting data from wing length measurements, daily adult survival assay, laboratory mating competition experiments, semi-field cage mating competition experiments, and semi-field cage swarm site attendance experiments. 


\section{Authors' contributions}

$A A, A P, L C, A P$ and $L C H$ contributed to the conception, design, and planning of experiments. LCH developed the field cage male swarming assay and generated the original DsRed line. AA and AP led experimental work and field collections in KPP, with assistance from KB, UK, WC, TF, NP, LJC and AP. LJC and $A Q$ ran nutritional analyses of mosquitoes and analysed data. Wing length measurements were coordinated by AP and AP. All other data was curated and analysed by AA. AA wrote the manuscript and produced all figures, with input and feedback from AP, AQ, LJC, AP and LCH. All authors read and approved the final manuscript.

\section{Funding}

AA, LCH and LCC were supported by BBSRC grants BB/N003594/1. Support for collection of field mosquitoes (conducted by the AFRIMS team) used in the DsRedKPP and KPPWT strains was provided by the Global Emerging Infections Surveillance (GEIS, U.S. Department of Defense) partner network.

\section{Availability of data and materials}

The datasets supporting the conclusions of this article are included within the article and its additional files.

\section{Ethics approval and consent to participate}

Use of animal blood to feed mosquitoes during the development of the DsRedKPP strain was approved through Imperial College London's Ethics Committee. Investigators provided study information and obtained the verbal consent from all participants. All procedures and study activities were reviewed and approved by Armed Forces Research Institute of Medical Sciences (Thailand) prior to commencement.

\section{Consent for publication}

Consent for publishing Fig. 1b was obtained from the subject.

\section{Competing interests}

The authors declare that they have no competing interests.

\section{Author details}

${ }^{1}$ Department of Life Sciences, Imperial College London, Silwood Park, Ascot, UK. ${ }^{2}$ Vector Biology and Control Section, Department of Entomology, Armed Forces Research Institute of Medical Sciences, Bangkok, Thailand. ${ }^{3}$ Department of Medical Entomology, Faculty of Tropical Medicine, Mahidol University, Bangkok, Thailand. ${ }^{4}$ Department of Entomology, Cornell University, Ithaca, NY, USA.

\section{Received: 5 April 2019 Accepted: 13 July 2019}

Published online: 19 July 2019

\section{References}

1. Fredericks AC, Fernandez-Sesma A. The burden of dengue and chikungunya worldwide: implications for the southern United States and California. Ann Glob Health. 2015;80:466

2. Benelli $\mathrm{G}$, Mehlhorn $\mathrm{H}$. Declining malaria, rising of dengue and Zika virus: insights for mosquito vector control. Parasitol Res. 2016;115:1747-54.

3. Alphey L, McKemey A, Nimmo D, Neira Oviedo M, Lacroix R, Matzen K, et al. Genetic control of Aedes mosquitoes. Pathog Glob Health. 2013:107:170-9.

4. McGraw EA, O'Neill SL. Beyond insecticides: new thinking on an ancient problem. Nat Rev Microbiol. 2013;11:181-93.

5. Carvalho DO, McKemey AR, Garziera L, Lacroix R, Donnelly CA, Alphey $L$, et al. Suppression of a field population of Aedes aegypti in Brazil by sustained release of transgenic male mosquitoes. PLoS Negl Trop Dis. 2015;9:1-15.

6. Lacroix R, McKemey AR, Raduan N, Kwee Wee L, Hong Ming W, Guat Ney T, et al. Open field release of genetically engineered sterile male Aedes aegypti in Malaysia. PLoS ONE. 2012;7:e42771.

7. O'Neill SL, Ryan PA, Turley AP, Wilson G, Retzki K, Iturbe-Ormaetxe I, et al. Scaled deployment of Wolbachia to protect the community from Aedes transmitted arboviruses. Gates Open Res. 2018;2:36.

8. Schmidt TL, Barton NH, Rašić G, Turley AP, Montgomery BL, IturbeOrmaetxe I, et al. Local introduction and heterogeneous spatial spread of dengue-suppressing Wolbachia through an urban population of Aedes aegypti. PLoS Biol. 2017;15:e2001894.

9. Lees RS, Gilles JR, Hendrichs J, Vreysen MJ, Bourtzis K. Back to the future: the sterile insect technique against mosquito disease vectors. Curr Opin Insect Sci. 2015;10:156-62.

10. Ferguson $\mathrm{H}$, John $\mathrm{B}, \mathrm{Ng}^{\prime}$ habi K, Knols B. Redressing the sex imbalance in knowledge of vector biology. Trends Ecol Evol. 2005:20:202-9.

11. Benelli G. Research in mosquito control: current challenges for a brighter future. Parasitol Res. 2015;114:2801-5.

12. Lees RS, Knols B, Bellini R, Benedict MQ, Bheecarry A, Bossin $H C$, et al. Review: improving our knowledge of male mosquito biology in relation to genetic control programmes. Acta Trop. 2014;132:S2-11.

13. Oliva CF, Damiens D, Benedict MQ. Male reproductive biology of Aedes mosquitoes. Acta Trop. 2014;132:S12-9.

14. Helinski MEH, Harrington LC. Considerations for male fitness in successful genetic vector control programs. In: Ecology of parasite-vector interactions. Wageningen: Wageningen Academic Publishers; 2013. p. 221-44.

15. Clements AN. The biology of mosquitoes, Volume 1: development, nutrition and reproduction. Wallingford: CABI; 1992.

16. Jansen CC, Beebe NW. The dengue vector Aedes aegypti: what comes next. Microbes Infect. 2010;12:272-9.

17. Bara JJ, Clark TM, Remold SK. Utilization of larval and pupal detritus by Aedes aegypti and Aedes albopictus. J Vector Ecol. 2014;39:44-7.

18. Schneider JR, Morrison AC, Astete H, Scott TW, Wilson ML. Adult size and distribution of Aedes aegypti (Diptera: Culicidae) associated with larval habitats in Iquitos, Peru. J Med Entomol. 2004:41:634-42.

19. Arrivillaga J, Barrera R. Food as a limiting factor for Aedes aegypti in water-storage containers. J Vector Ecol. 2004;29:11-20.

20. Koenraadt CJM, Kormaksson M, Harrington LC. Effects of inbreeding and genetic modification on Aedes aegypti larval competition and adult energy reserves. Parasit Vectors. 2010;3:92.

21. Kingsolver JG, Huey RB. Size, temperature, and fitness: three rules. Evol Ecol Res. 2008;10:251-68.

22. Padmanabha H, Bolker B, Lord CC, Rubio C, Lounibos LP. Food availability alters the effects of larval temperature on Aedes aegypti growth. J Med Entomol. 2011;48:974-84.

23. Helinski MEH, Harrington LC. Male mating history and body size influence female fecundity and longevity of the dengue vector Aedes aegypti. J Med Entomol. 2011;48:202-11.

24. Briegel $\mathrm{H}$. Metabolic relationship between female body size, reserves, and fecundity of Aedes aegypti. J Insect Physiol. 1990;36:165-72.

25. Ponlawat A, Harrington LC. Age and body size influence male sperm capacity of the dengue vector Aedes aegypti (Diptera: Culicidae). J Med Entomol. 2007;44:422-6.

26. Ponlawat A, Harrington LC. Factors associated with male mating success of the dengue vector mosquito, Aedes aegypti. Am J Trop Med Hyg. 2009;80:395-400

27. Maciel-De-Freitas R, Codeço CT, Lourenço-De-Oliveira R. Body sizeassociated survival and dispersal rates of Aedes aegypti in Rio de Janeiro. Med Vet Entomol. 2007:21:284-92.

28. Lang BJ, Idugboe S, McManus K, Drury F, Qureshi A, Cator LJ. The effect of larval diet on adult survival, swarming activity and copulation success in male Aedes aegypti (Diptera: Culicidae). J Med Entomol. 2018:55:29-35

29. Coon KL, Vogel KJ, Brown MR, Strand MR. Mosquitoes rely on their gut microbiota for development. Mol Ecol. 2014;23:2727-39.

30. Coon KL, Brown MR, Strand MR. Mosquitoes host communities of bacteria that are essential for development but vary greatly between local habitats. Mol Ecol. 2016:25:5806-26.

31. Strand MR. Composition and functional roles of the gut microbiota in mosquitoes. Curr Opin Insect Sci. 2018;28:59-65.

32. Dickson LB, Jiolle D, Minard G, Moltini-Conclois I, Volant S, Ghozlane A, et al. Carryover effects of larval exposure to different environmental bacteria drive adult trait variation in a mosquito vector. Sci Adv. 2017;3:1-15.

33. Coon KL, Brown MR, Strand MR. Gut bacteria differentially affect egg production in the anautogenous mosquito Aedes aegypti and facultatively autogenous mosquito Aedes atropalpus (Diptera: Culicidae). Parasit Vectors. 2016:9:375 
34. Kang DS, Alcalay Y, Lovin DD, Cunningham JM, Eng MW, Chadee DD, et al. Larval stress alters dengue virus susceptibility in Aedes aegypti (L.) adult females. Acta Trop. 2017;174:97-101.

35. Evans MV, Shiau JC, Solano N, Brindley MA, Drake JM, Murdock CC. Carryover effects of urban larval environments on the transmission potential of dengue-2 virus. Parasit Vectors. 2018;11:426.

36. Moller-Jacobs LL, Murdock CC, Thomas MB. Capacity of mosquitoes to transmit malaria depends on larval environment. Parasit Vectors. 2014;7:593.

37. Takken W, Smallegange RC, Vigneau AJ, Johnston V, Brown M, MordueLuntz AJ, et al. Larval nutrition differentially affects adult fitness and Plasmodium development in the malaria vectors Anopheles gambiae and Anopheles stephensi. Parasit Vectors. 2013;6:345.

38. Okech BA, Gouagna LC, Yan G, Githure JI, Beier JC. Larval habitats of Anopheles gambiae s.s. (Diptera: Culicidae) influences vector competence to Plasmodium falciparum parasites. Malar J. 2007;6:50.

39. Vantaux A, Lefèvre T, Cohuet A, Dabiré KR, Roche B, Roux O, et al. Larval nutritional stress affects vector life history traits and human malaria transmission. Sci Rep. 2016;6:36778.

40. Gendrin M, Christophides GK. The Anopheles mosquito microbiota and their impact on pathogen transmission. In: Anopheles mosquitoes-new insights into malaria vectors. Rijeka: InTech; 2013. p. 137-64.

41. Dennison NJ, Jupatanakul N, Dimopoulos G. The mosquito microbiota influences vector competence for human pathogens. Curr Opin Insect Sci. 2014;3:6-13.

42. Hegde S, Rasgon JL, Hughes GL. The microbiome modulates arbovirus transmission in mosquitoes. Curr Opin Virol. 2015;15:97-102.

43. Lewis Z, Lizé A. Insect behaviour and the microbiome. Curr Opin Insect Sci. 2015:9:86-90

44. Callahan AG, Ross PA, Hoffmann AA. Small females prefer small males: size assortative mating in Aedes aegypti mosquitoes. Parasit Vectors. 2018;11:445.

45. Cator LJ, Zanti Z. Size, sounds and sex: interactions between body size and harmonic convergence signals determine mating success in Aedes aegypti. Parasit Vectors. 2016;9:622.

46. Yeap HL, Endersby NM, Johnson PH, Ritchie SA, Hoffmann AA. Body size and wing shape measurements as quality indicators of Aedes aegypti mosquitoes destined for field release. Am JTrop Med Hyg. 2013;89:78-92.

47. Ami EB, Yuval B, Jurkevitch E. Manipulation of the microbiota of massreared Mediterranean fruit flies Ceratitis capitata (Diptera: Tephritidae) improves sterile male sexual performance. ISME J. 2010;4:28-37.

48. Gavriel S, Jurkevitch E, Gazit Y, Yuval B. Bacterially enriched diet improves sexual performance of sterile male Mediterranean fruit flies. J Appl Entomol. 2011;135:564-73.

49. Cai Z, Yao Z, Li Y, Xi Z, Bourtzis K, Zhao Z, et al. Intestinal probiotics restore the ecological fitness decline of Bactrocera dorsalis by irradiation. Evol Appl. 2018;11:1946-63.

50. Sharon G, Segal D, Ringo JM, Hefetz A, Zilber-Rosenberg I, Rosenberg E. Commensal bacteria play a role in mating preference of Drosophila melanogaster. Proc Natl Acad Sci USA. 2010;107:20051-6.

51. Arbuthnott $D$, Levin TC, Promislow DEL. The impacts of Wolbachia and the microbiome on mate choice in Drosophila melanogaster. J Evol Biol. 2016:29:461-8.

52. Pike A, Dong Y, Dizaji NB, Gacita A, Mongodin EF, Dimopoulos G. Changes in the microbiota cause genetically modified Anopheles to spread in a population. Science. 2017;357:1396-9.

53. Leftwich PT, Bolton M, Chapman T. Evolutionary biology and genetic techniques for insect control. Evol Appl. 2016;9:212-30.

54. Ross PA, Endersby-Harshman NM, Hoffmann AA. A comprehensive assessment of inbreeding and laboratory adaptation in Aedes aegypti mosquitoes. Evol Appl. 2019;12:572-86.

55. Soma DD, Maïga H, Mamai W, Bimbile-Somda NS, Venter N, Ali AB, et al. Does mosquito mass-rearing produce an inferior mosquito? Malar J. 2017;16:357.

56. Bargielowski I, Alphey L, Koella JC. Cost of mating and insemination capacity of a genetically modified mosquito Aedes aegypti OX513A compared to its wild type counterpart. PLoS ONE. 2011;6:e26086.

57. De Jesus $C E$, Reiskind $M H$. The importance of male body size on sperm uptake and usage, and female fecundity in Aedes aegypti and Aedes albopictus. Parasit Vectors. 2016;9:447.
58. Massonnet-Bruneel B, Corre-Catelin N, Lacroix R, Lees RS, Hoang KP, Nimmo D, et al. Fitness of transgenic mosquito Aedes aegypti males carrying a dominant lethal genetic system. PLoS ONE. 2013;8:e62711.

59. Patil PB, Niranjan Reddy BP, Gorman K, Seshu Reddy KV, Barwale SR, Zehr UB, et al. Mating competitiveness and life-table comparisons between transgenic and Indian wild-type Aedes aegypti L. Pest Manag Sci. 2015;71:957-65.

60. Facchinelli L, Valerio L, Ramsey JM, Gould F, Walsh RK, Bond G, et al. Field cage studies and progressive evaluation of genetically-engineered mosquitoes. PLoS Negl Trop Dis. 2013;7:e2001.

61. Segoli M, Hoffmann AA, Lloyd J, Omodei GJ, Ritchie SA. The effect of virus-blocking Wolbachia on male competitiveness of the dengue vector mosquito, Aedes aegypti. PLoS Negl Trop Dis. 2014;8:e3294.

62. Benedict MQ, Knols BGJ, Bossin HC, Howell PI, Mialhe E, Caceres C, et al. Colonisation and mass rearing: learning from others. Malar J. 2009;8:S4.

63. Baeshen R, Ekechukwu NE, Toure M, Paton D, Coulibaly M, Traoré SF, et al. Differential effects of inbreeding and selection on male reproductive phenotype associated with the colonization and laboratory maintenance of Anopheles gambiae. Malar J. 2014;13:19.

64. Thai Ministry of Public Health. http://www.boe.moph.go.th. Accessed 10 Feb 2019.

65. Mammen MP, Pimgate C, Koenraadt CJM, Rothman AL, Aldstadt J, Nisalak A, et al. Spatial and temporal clustering of dengue virus transmission in Thai villages. PLoS Med. 2008;5:1605-16.

66. Thai Meteorological Department. https://www.tmd.go.th. Accessed 10 Feb 2019.

67. Rattanarithikul R, Panthusiri P. Illustrated keys to the medically important mosquitos of Thailand. Southeast Asian J Trop Med Public Health. 1994:25(Suppl. 1):1-66.

68. Smith RC, Walter MF, Hice RH, O'Brochta DA, Atkinson PW. Testis-specific expression of the $\beta 2$ tubulin promoter of Aedes aegypti and its application as a genetic sex-separation marker. Insect Mol Biol. 2007;16:61-71.

69. Van Handel E. Rapid determination of glycogen and sugars in mosquitoes. J Am Mosq Control Assoc. 1985;1:299-301.

70. Van Handel E. Rapid determination of total lipids in mosquitoes. J Am Mosq Control Assoc. 1985;1:302-4.

71. Van Handel E, Day JF. Assay of lipids, glycogen and sugars in individual mosquitoes: correlations with wing length in field-collected Aedes vexans. J Am Mosq Control Assoc. 1988;4:549-50.

72. Kaufmann C, Brown MR. Regulation of carbohydrate metabolism and flight performance by a hypertrehalosaemic hormone in the mosquito Anopheles gambiae. J Insect Physiol. 2008:54:367-77.

73. Dickens BL, Brant HL. Effects of marking methods and fluorescent dusts on Aedes aegypti survival. Parasit Vectors. 2014;7:65.

74. Nasci RS. Relationship of wing length to adult dry weight in several mosquito species (Diptera: Culicidae). J Med Entomol. 1990;27:716-9.

75. Vazquez-Prokopec GM, Galvin WA, Kelly R, Kitron U. A new, cost-effective, battery-powered aspirator for adult mosquito collections. J Med Entomol. 2009:46:1256-9.

76. R Core Team. R: a language and environment for statistical computing. Vienna: R Foundation for Statistical Computing; 2016.

77. Fox J, Weisberg S. An R companion to applied regression. 2nd ed. Thousand Oaks: Sage; 2011.

78. Lenth RV. Least-squares means: $t R$ package Ismeans. J Stat Softw. 2016;69:1.

79. Bates D, Mächler M, Bolker B, Walker S. Fitting linear mixed-effects models using Ime4. J Stat Softw. 2015;67:1.

80. Hothorn T, Bretz F, Westfall P. Simultaneous inference in general parametric models. Biom J. 2008;50:346-63.

81. Wickham H, Francois R, Henry L, Müller K. dplyr: a grammar of data manipulation. R package version 0.7.4; 2017.

82. Therneau TM. coxme: mixed effects Cox models. R Package version 2.2-7; 2018.

83. Harrington DP, Fleming TR. A class of rank test procedures for censored survival data. Biometrika. 1982;69:553.

84. Ross PA, Axford JK, Richardson KM, Endersby-Harshman NM, Hoffmann AA. Maintaining Aedes aegypti mosquitoes infected with Wolbachia. J Vis Exp. 2017;126:e56124.

85. Dickson LB, Ghozlane A, Volant S, Bouchier C, Ma L, Vega-Rúa A, et al. Diverse laboratory colonies of Aedes aegypti harbor the same adult midgut bacterial microbiome. Parasit Vectors. 2018;11:207. 
86. Sawadogo SP, Diabaté A, Toé HK, Sanon A, Lefevre T, Baldet T, et al. Effects of age and size on Anopheles gambiae s.s. male mosquito mating success. J Med Entomol. 2013;50:285-93.

87. Maïga H, Dabiré RK, Lehmann T, Tripet F, Diabaté A. Variation in energy reserves and role of body size in the mating system of Anopheles gambiae. J Vector Ecol. 2012;37:289-97.

88. Han CS, Jablonski PG, Kim B, Park FC. Size-assortative mating and sexual size dimorphism are predictable from simple mechanics of mate-grasping behavior. BMC Evol Biol. 2010;10:359.

89. Aldersley A, Cator LJ. Female resistance and harmonic convergence influence male mating success in Aedes aegypti. Sci Rep. 2019;9:2145.

90. Qureshi A, Aldersley A, Hollis B, Ponlawat A, Cator L. Male competition and the evolution of mating and life history traits in experimental populations of Aedes aegypti. Proc R Soc B Biol Sci. 2019;286:20190591.

91. Helinski MEH, Valerio L, Facchinelli L, Scott TW, Ramsey J, Harrington LC. Evidence of polyandry for Aedes aegypti in semifield enclosures. Am J Trop Med Hyg. 2012;86:635-41.

92. Reisen WK, Milby MM, Asman SM, Bock ME, Meyer RP, McDonald PT, et al. Attempted suppression of a semi-isolated Culex tarsalis population by the release of irradiated males: a second experiment using males from a recently colonized strain. Mosq News. 1982;42:565-75.

93. Oliva CF, Jacquet M, Gilles J, Lemperiere G, Maquart PO, Quilici S, et al. The sterile insect technique for controlling populations of Aedes albopictus (Diptera: Culicidae) on Reunion Island: mating vigour of sterilized males. PLOS ONE. 2012;7:e49414.
94. Chambers EW, Hapairai L, Peel BA, Bossin H, Dobson SL. Male mating competitiveness of a Wolbachia-introgressed Aedes polynesiensis strain under semi-field conditions. PLoS Negl Trop Dis. 2011;5:e1271.

95. Bellini R, Balestrino F, Medici A, Gentile G, Veronesi R, Carrieri M. Mating competitiveness of Aedes albopictus radio-sterilized males in large enclosures exposed to natural conditions. J Med Entomol. 2013;50:94-102.

96. Bourtzis K, Lees RS, Hendrichs J, Vreysen MJB. More than one rabbit out of the hat: radiation, transgenic and symbiont-based approaches for sustainable management of mosquito and tsetse fly populations. Acta Trop. 2016;157:115-30.

97. Culbert NJ, Balestrino F, Dor A, Herranz GS, Yamada H, Wallner T, et al. A rapid quality control test to foster the development of genetic control in mosquitoes. Sci Rep. 2018;8:16179.

98. Madakacherry O, Lees RS, Gilles JRL. Aedes albopictus (Skuse) males in laboratory and semi-field cages: release ratios and mating competitiveness. Acta Trop. 2014;132:S124-9.

99. Ponlawat A, Harrington LC. Blood feeding patterns of Aedes aegypti and Aedes albopictus in Thailand. J Med Entomol. 2005;42:844-9.

\section{Publisher's Note}

Springer Nature remains neutral with regard to jurisdictional claims in published maps and institutional affiliations.
Ready to submit your research? Choose BMC and benefit from:

- fast, convenient online submission

- thorough peer review by experienced researchers in your field

- rapid publication on acceptance

- support for research data, including large and complex data types

- gold Open Access which fosters wider collaboration and increased citations

- maximum visibility for your research: over 100M website views per year

At BMC, research is always in progress.

Learn more biomedcentral.com/submissions 